\title{
Exponential Dichotomy and Bifurcation Conditions of Solutions of the Hamiltonian Operators Boundary Value Problems in the Hilbert Space
}

\author{
Pokutnyi Oleksandr \\ Institute of Mathematics, National Academy of Sciences of Ukraine, Kiev, 01004, Ukraine
}

Copyright (C) 2018 by authors, all rights reserved. Authors agree that this article remains permanently open access under the terms of the Creative Commons Attribution License 4.0 International License

\begin{abstract}
Sufficient conditions for the existence of solutions for a weakly linear perturbed boundary value problem are obtained in the so called resonance (critical) case. Iterative process for finding solutions has been presented. Necessary and sufficient conditions of the existence of solutions, bounded solutions, generalized solutions and quasi solutions are obtained.
\end{abstract}

Keywords Bifurcation Conditions, Lyapunov Equation, Exponential Dichotomy, Vishik-Lyusternik Method

\section{Introduction}

Methods of perturbation theory, whose foundations were laid by Poincare and Lyapunov, are a powerful tool in applied mathematics, mechanics and permit one to obtain approximate analytical representations of solutions of rather complicated boundary value problems. Most of these methods arose when solving specific problems of mechanics, celestial mechanics, and physics [1]-[8]. Numerous examples of various problems that can be studied by operator methods of perturbation theory can be found in the monograph [6]. The present paper uses the theory of generalized inverse and Moore-Penrose pseudoinverse operators [9] - [13] to construct perturbation theory for the Lyapunov type equation [14], [15] in resonance cases [16]. We investigate bifurcation conditions of solutions of boundary value problems in the Hilbert space when considering problem has the set of solutions not for any right hand side of the equation.

We use the modification of the well-known VishikLyusternik [17] method on the case of operator differential boundary value problems.

Statement of the problem. Consider boundary value problem

$$
\frac{d X(t, \varepsilon)}{d t}=[A, X(t, \varepsilon)]+\Phi(t)+\varepsilon D(t) X(t, \varepsilon), t \in J
$$

$$
l X(\cdot, \varepsilon)=\alpha .
$$

Here $X(t, \varepsilon)$ is an unknown operator-valued function from the space $C^{1}(J, \mathcal{L}(H))$.

$$
\begin{gathered}
C^{1}(J, \mathcal{L}(H)):=\{X: J \rightarrow \mathcal{L}(H), \\
\left.\|X\|=\sup _{t \in J} \max \left\{\|X(t)\|,\left\|\frac{d X(t)}{d t}\right\|\right\}<\infty\right\}
\end{gathered}
$$

is the space of continuously differentiable operator-valued functions (or another function spaces), $J \subset R ; A \in \mathcal{L}(H)$ is a linear and bounded operator

$$
[A, X(t)]=A X(t)-X(t) A
$$

$D(t), \Phi(t) \in C(J, \mathcal{L}(H))$ are given strongly-continuous operator-valued functions; $l: C^{1}(J, \mathcal{L}(H)) \rightarrow H_{1}$ is linear and bounded operator which translates solutions of (1) into the Hilbert space $H_{1}$. At first we seek the solution of boundary value problem $(1,2)$ which for $\varepsilon=0$ turns in one of the solutions of generating problem $(3,4)$

$$
\begin{gathered}
\frac{d X(t)}{d t}=[A, X(t)]+\Phi(t), \\
l X(\cdot, \varepsilon)=\alpha .
\end{gathered}
$$

We find solution $X(t, \varepsilon) \in C^{1}(J ; \mathcal{L}(H)) \times C\left(0, \varepsilon_{0}\right]$ for a fixed $\varepsilon_{0}>0(J \subset[a ; b])$.

\section{Unperturbed equation}

1) Consider the case when generating problem has solutions [18], [19]. We find the solution of boundary value problem (1), (2) in the form of a series

$$
X(t, \varepsilon)=\sum_{i=0}^{+\infty} \varepsilon^{i} X_{i}(t) .
$$


Equating coefficients under corresponding powers of $\varepsilon$ for $\varepsilon^{0}$ we obtain the following boundary value problem

$$
\begin{gathered}
\frac{d X_{0}(t)}{d t}=\left[A, X_{0}(t)\right]+\Phi(t), \\
l X_{0}(\cdot)=\alpha .
\end{gathered}
$$

Solutions of the equation (5) have the following form:

$$
X_{0}(t, M)=e^{t A} M e^{-t A}+\int_{0}^{t} e^{(t-\tau) A} \Phi(\tau) e^{(\tau-t) A} d \tau,
$$

for every operator $M \in \mathcal{L}(H)$. Really

$$
\begin{aligned}
& \frac{d X_{0}(t, M)}{d t}=A e^{t A} M e^{-t A}-e^{t A} M e^{-t A} A+\Phi(t)+ \\
& +A \int_{0}^{t} e^{(t-\tau) A} \Phi(\tau) e^{(\tau-t) A} d \tau- \\
& -\int_{0}^{t} e^{(t-\tau) A} \Phi(\tau) e^{(\tau-t) A} d \tau A= \\
& =A X_{0}(t, M)-X_{0}(t, M) A+\Phi(t) . \\
& \left(\int_{0}^{t} e^{(t-\tau) A} \Phi(\tau) e^{(\tau-t) A} d \tau\right)_{t}^{\prime}=e^{(t-\tau) A} \Phi(t) e^{(t-\tau) A}+ \\
& +\int_{0}^{t}\left(e^{(t-\tau) A} \Phi(\tau) e^{(\tau-t) A}\right)_{t}^{\prime} d \tau= \\
& =\Phi(t)+\int_{0}^{t}\left(e^{(t-\tau) A}\right)_{t}^{\prime} \Phi(\tau) e^{(\tau-t) A} d \tau+ \\
& +\int_{0}^{t} e^{(t-\tau) A} \Phi(\tau)\left(e^{(\tau-t) A}\right)_{t}^{\prime} d \tau= \\
& =\Phi(t)+\int_{0}^{t} A e^{(t-\tau) A} \Phi(\tau) e^{(\tau-t) A} d \tau- \\
& -\int_{0}^{t} e^{(t-\tau) A} \Phi(\tau) e^{(\tau-t) A} A d \tau= \\
& =\Phi(t)+A \int_{0}^{t} e^{(t-\tau) A} \Phi(\tau) e^{(\tau-t) A} d \tau- \\
& -\int_{0}^{t} e^{(t-\tau) A} \Phi(\tau) e^{(\tau-t) A} d \tau A .
\end{aligned}
$$

Substituting in the boundary condition (6) we obtain the following operator equation

$$
Q M=g_{0},
$$

where

$$
\begin{gathered}
Q M=l e^{\cdot A} M e^{-\cdot A}, \\
g_{0}=\alpha-l \int_{0}^{\cdot} e^{(\cdot-\tau) A} \Phi(\tau) e^{(\tau-\cdot) A} d \tau .
\end{gathered}
$$

Let us study the solvability of the operator equation (8) in the Hilbert spaces. We distinguish three types of solutions.
1. Classical solutions. If the operator $Q$ is normally solvable, i.e., has closed range $(R(Q)=\overline{R(Q)})$, then it is well known [11] that $g \in R(Q)$ if and only if $P_{N\left(Q^{*}\right)} g_{0}=0$, where $P_{N\left(Q^{*}\right)}$ is the projection onto the cokernel of $Q$ [11]. In this case, there exists a generalized inverse $Q^{-}[11]$ and the solution set of equation (8) can be represented in the form

$$
M=Q^{-} g_{0}+P_{N(Q)} M_{0}, M_{0} \in \mathcal{L}(H),
$$

where $P_{N(Q)}$ is the projection onto the kernel of $Q$.

2. Strong generalized solutions. Now assume that the range of $Q$ is not closed, $R(Q) \neq \overline{R(Q)}$. Let us show that $Q$ admits a normally solvable extension $\bar{Q}, R(\bar{Q})=\overline{R(Q)}$. Since the operator $Q$ is linear and bounded, we have the following direct sum decomposition of the space $H_{1}$ :

$$
H_{1}=\overline{R(Q)} \oplus Y
$$

where $Y=\overline{R(Q)}^{\perp}$. Suppose that

$$
\mathcal{L}(H)=N(Q) \oplus X .
$$

By virtue of these decompositions, there exist projections $P_{N(Q)}, P_{X}$, orthogonal projections $\mathcal{P}_{\overline{R(Q)}}$, and $\mathcal{P}_{Y}$ onto the respective subspaces. Let $H_{2}=\mathcal{L}(H) / N(Q)$ be the quotient space of $\mathcal{L}(H)$ by the kernel $N(Q)$. It is well known [13], [20] that there exists a continuous bijection $p: X \rightarrow H_{2}$ and a projection $j: \mathcal{L}(H) \rightarrow H_{2}$. The triple $\left(\mathcal{L}(H), H_{2}, j\right)$ is a locally trivial bundle with fiber $P_{N(Q)} \mathcal{L}(H)$. Consider the operator

$$
\mathcal{Q}=\mathcal{P} \overline{R(Q)} Q j^{-1} p: X \rightarrow R(Q) \subset \overline{R(Q)} .
$$

One can readily verify that it is linear, injective, and continuous. Now we use completion [21] with respect to the norm $\|x\|_{\bar{X}}=\|\mathcal{Q} x\|_{F}$, where $F=\overline{R(Q)}$, and obtain a new space $\bar{X}$ and an extended operator $\overline{\mathcal{Q}}$. Then

$$
\overline{\mathcal{Q}}: \bar{X} \rightarrow \overline{R(Q)}, \quad X \subset \bar{X},
$$

and the operator thus constructed is a homeomorphism between $\bar{X}$ and $\overline{R(Q)}$. Consider the extended operator $\bar{Q}=$ $\overline{\mathcal{Q}} \mathcal{P}_{\bar{X}}: \overline{\mathcal{L}(H)} \rightarrow H_{1}$,

$$
\overline{\mathcal{L}(H)}=N(Q) \oplus \bar{X}, H_{1}=R(\bar{Q}) \oplus Y .
$$

Obviously, $\bar{Q} M=Q M, M \in \mathcal{L}(H)$, and the operator $\bar{Q}$ is normally solvable $(R(\bar{Q})=\overline{R(\bar{Q}))}$ and hence generalizedinvertible with generalized-inverse $\bar{Q}-$, which will be called the strong generalized-inverse of $Q$ [13], [20].

Now we use the operator $\bar{Q}^{-}$to establish the generalized solvability of equation (8). The criterion for the generalized solvability of equation (8) has the form

$$
\mathcal{P}_{Y} g_{0}=0,
$$

and any element of the set $\left\{\bar{Q}^{-} g_{0}+P_{N(Q)} M_{0}: M_{0} \in \mathcal{L}(H)\right\}$ will be called the corresponding strong generalized solution of equation (8).

Remark 1. i. Note that $P_{N(\bar{Q})}=P_{N(Q)}$ and the set $\left\{\bar{Q}^{-} g_{0}+P_{N(Q)} M_{0}: M_{0} \in \mathcal{L}(H)\right\}$ consists of usual solutions of the equation $\bar{Q} M=g_{0}$; hence the element $g_{0}$ belongs 
to the range of the operator $\bar{Q}$. If $g_{0} \in R(Q)$, then the strong generalized solution defined above is a classical solution.

ii. If we consider equality $Q M=g_{0}$ at every point $x \in H$ then for the representation of solutions we can use the MoorePenrose pseudo invertible operator $Q^{+}$or $\bar{Q}^{+}$.

3. Generalized quasi-solutions. Now consider the case in which $g_{0} \notin \overline{R(Q)}$, or, equivalently, the element $g_{0}$ satisfies the condition $\mathcal{P}_{Y} g_{0} \neq 0$. Then there exist no strong generalized solutions, but there exist elements of $\bar{X}$ that are solutions of the variational problem inf $\|\bar{Q} M-g\|_{\bar{H}_{1}}$, where $\bar{Q}=\bar{Q}_{X} \mathcal{P}_{\bar{X}}$ and the infimum is taken over all $M_{0} \in \bar{X}$. The set of these elements has the form $\left\{\bar{Q}^{-} g_{0}+P_{N(Q)} M_{0}: M_{0} \in \mathcal{L}(H)\right\}$. We call them generalized quasi-solutions by analogy with the usual quasi-solutions [11]. Thus, the following theorem holds.

Theorem 1. Boundary value problem (5), (6), in Hilbert spaces has:

1. Strong generalized solutions if and only if

$$
\mathcal{P}_{Y}\left\{\alpha-l \int_{0}^{\cdot} e^{(\cdot-\tau) A} \Phi(\tau) e^{(\tau-\cdot) A} d \tau\right\}=0
$$

\section{Generalized quasi-solutions if and only if}

$$
\mathcal{P}_{Y}\left\{\alpha-l \int_{0}^{\cdot} e^{(\cdot-\tau) A} \Phi(\tau) e^{(\tau-\cdot) A} d \tau\right\} \neq 0
$$

3. Under condition (9) or (10), the strong generalized solutions, classical solutions, and quasi-solutions of the boundary value problem (5), (6) have the form

$X_{0}\left(t, M_{0}\right)=e^{t A} P_{N(Q)} M_{0} e^{-t A}+e^{t A} \bar{Q}^{-} \alpha e^{-t A}+\overline{(G[\Phi])}(t)$,

where

$$
\begin{gathered}
\overline{(G[\Phi])}(t)=\int_{0}^{t} e^{(t-\tau) A} \Phi(\tau) e^{(\tau-t) A} d \tau- \\
-l \int_{0}^{\cdot} e^{(\cdot-\tau) A} \Phi(\tau) e^{(\tau-\cdot) A} d \tau
\end{gathered}
$$

is the generalized Green operator of the boundary value problem (5), (6) and $M_{0}$ is an arbitrary operator of $\mathcal{L}(H)$.

Remark 2. If the range of $Q$ is closed, i.e., the operator $Q$ is normally solvable, then condition (9) guarantees the existence of classical solutions. In this case, it is equivalent to the condition

$$
\alpha-l \int_{0}^{\cdot} e^{(\cdot-\tau) A} \Phi(t) e^{(\tau-\cdot) A} d \tau \in R(Q) .
$$

Remark 3. If the operator $A$ and operator-valued function $X(t, \varepsilon)$ are commute $[A, X(t, \varepsilon)]=0$ then we obtain the following boundary value problem

$$
\begin{gathered}
X^{\prime}(t, \varepsilon)=\Phi(t)+\varepsilon D(t) X(t, \varepsilon), \\
l X(\cdot, \varepsilon)=\alpha .
\end{gathered}
$$

Generating boundary value problem has the following form

$$
X^{\prime}(t, \varepsilon)=\Phi(t),
$$

$$
l X(\cdot, \varepsilon)=\alpha .
$$

Due to the theorem 1 we obtain the following corollary. (In this case $Q=l$ ).

Corollary. Boundary value problem (14), (15), in Hilbert spaces has:

1. Strong generalized solutions if and only if

$$
\mathcal{P}_{Y}\left\{\alpha-l \int_{0}^{\cdot} \Phi(\tau) d \tau\right\}=0
$$

2. Generalized quasi-solutions if and only if

$$
\mathcal{P}_{Y}\left\{\alpha-l \int_{0}^{\cdot} \Phi(\tau) d \tau\right\} \neq 0
$$

3. Under condition (16) or (17), the strong generalized solutions, classical solutions, and quasi-solutions of the boundary value problem (14), (15) have the form

$X_{0}(t, C)=P_{N(Q)} C+\bar{Q}^{-}\left\{\alpha-l \int_{0}^{\cdot} \Phi(\tau) d \tau\right\}+\int_{0}^{t} \Phi(\tau) d \tau$,

$C \in \mathcal{L}(H)$. We can see that in the case when $[A, X(t, \varepsilon)]=0$ we don't get a trivial situation.

\section{Bifurcation condition}

Assume that the boundary value problem (3), (4) ((5), (6)) has classical solutions; i.e., condition (9) is satisfied. Let us find conditions on the operators $D(t)$ and $l$ under which the perturbed boundary value problem (1), (2) has classical solutions. Let us show that this problem can be solved with the use of the operator

$$
B_{0} M=\mathcal{P}_{Y} l \int_{0}^{\cdot} e^{(\cdot-\tau) A} D(\tau) e^{\tau A} \mathcal{P}_{N(Q)} M e^{-\tau A} e^{(\tau-\cdot) A} d \tau
$$

As we say eariler, following [22], we seek solutions of the boundary value problem (1), (2) in the form of the series

$$
X(t, \varepsilon)=\sum_{i=0}^{+\infty} \varepsilon^{i} X_{i}(t)
$$

in powers of the small parameter $\varepsilon$. Operator $M_{0} \in \mathcal{L}(H)$ will be determined at the next stage of the iterative process. The coefficient $X_{1}(t)$ of $\varepsilon^{1}$ is a solution of the boundary value problem

$$
\begin{gathered}
\frac{d X_{1}(t)}{d t}=\left[A, X_{1}(t)\right]+D(t) X_{0}\left(t, M_{0}\right), \\
l X_{1}(\cdot)=0 .
\end{gathered}
$$

In view of condition (9), the solvability criterion of problem (21), (22) acquires the form

$$
-\mathcal{P}_{Y} l \int_{0}^{\cdot} e^{(\cdot-\tau) A} D(\tau) X_{0}\left(\tau, M_{0}\right) e^{(\tau-\cdot) A} d \tau=0,
$$

whence we finally obtain the operator equation

$$
B_{0} M_{0}=-\mathcal{P}_{Y}\left[l \int _ { 0 } ^ { \cdot } e ^ { ( \cdot - \tau ) A } D ( \tau ) \left\{e^{\tau A} \bar{Q}^{-} \alpha e^{-\tau}+\right.\right.
$$




$$
\left.+\overline{(G[\Phi])}(\tau)\} e^{(\tau-\cdot) A} d \tau\right] .
$$

For simplicity suppose that the operator $B_{0}$ is generalized invertible and $\mathcal{P}_{N\left(B_{0}^{*}\right)} \mathcal{P}_{Y}=0$, where $\mathcal{P}_{N\left(B_{0}^{*}\right)}$ is the projection onto the kernel of adjoint operator $B_{0}^{*}$. Then equation (24) is solvable. The set of strong generalized solutions of equation (24) has the form

$$
\begin{aligned}
M_{0}=- & B_{0}^{-} \mathcal{P}_{Y}\left[l \int _ { 0 } ^ { \cdot } e ^ { ( \cdot - \tau ) A } D ( \tau ) \left\{e^{\tau A} \bar{Q}^{-} \alpha e^{-\tau A}+\right.\right. \\
& \left.+\overline{(G[\Phi])}(\tau)\} e^{(\tau-\cdot) A} d \tau\right]+P_{N\left(B_{0}\right)} M_{\rho}
\end{aligned}
$$

where $M_{\rho} \in \mathcal{L}(H)$ is arbitrary, $P_{N\left(B_{0}\right)}$ is a projection onto the kernel of operator $B_{0}$. For convenience, we rewrite this relation in the form

$$
M_{0}=\bar{M}_{0}+P_{N\left(B_{0}\right)} M_{\rho}
$$

where

$$
\begin{gathered}
\bar{M}_{0}=-B_{0}^{-} \mathcal{P}_{Y}\left[l \int _ { 0 } ^ { \cdot } e ^ { ( \cdot - \tau ) A } D ( \tau ) \left\{e^{\tau A} \bar{Q}^{-} \alpha e^{-\tau A}+\right.\right. \\
\left.+\overline{(G[\Phi])}(\tau)\} e^{(\tau-\cdot) A} d \tau\right] .
\end{gathered}
$$

Then the solution set of the boundary value problem (5), (6) has the form

$$
X_{0}\left(t, M_{\rho}\right)=\bar{X}_{0}\left(t, \bar{M}_{0}\right)+\bar{Y}_{0}(t)\left[P_{N\left(B_{0}\right)} M_{\rho}\right], M_{\rho} \in \mathcal{L}(H)
$$

$\bar{X}_{0}\left(t, \bar{M}_{0}\right)=e^{t A} P_{N(Q)} \bar{M}_{0} e^{-t A}+e^{t A} \bar{Q}^{-} \alpha e^{-t A}+\overline{G[\Phi]}(t)$, operator $\bar{Y}_{0}$ is defined by the rule

$$
\bar{Y}_{0}(t)[R]:=e^{t A} P_{N(Q)} R e^{-t A} .
$$

Now we use the linearity of the generalized Green operator to represent the solution set of the boundary value problem in the form

$$
X_{1}\left(t, M_{1}\right)=e^{t A} P_{N(Q)} M_{1} e^{-t A}+\overline{\left(G\left[D(\cdot) X_{0}\left(\cdot, M_{\rho}\right)\right]\right)}(t),
$$

or

$$
\begin{aligned}
X_{1}\left(t, M_{1}\right)= & e^{t A} P_{N(Q)} M_{1} e^{-t A}+\overline{\left(G\left[D(\cdot) \bar{X}_{0}\left(\cdot, \bar{M}_{0}\right)\right]\right)}(t)+ \\
& +\overline{\left(G\left[D(\cdot) \bar{Y}_{0}(\cdot)\left[P_{N\left(B_{0}\right)} M_{\rho}\right)\right]\right)}(t),
\end{aligned}
$$

where the operator $M_{1}$ will be found at the next step of the iterative process. The coefficient $X_{2}(t)$ of $\varepsilon^{2}$ in the series (20) is a solution of the boundary value problem

$$
\begin{gathered}
\frac{d X_{2}(t)}{d t}=\left[A, X_{2}(t)\right]+D(t) X_{1}\left(t, M_{1}\right), \\
l X_{2}(\cdot)=0 .
\end{gathered}
$$

The solvability condition (9) for the problem (26), (27) becomes

$$
-\mathcal{P}_{Y} l \int_{0}^{\cdot} e^{(\cdot-\tau) A} D(\tau) X_{1}\left(\tau, M_{1}\right) e^{(\tau-\cdot) A} d \tau=0
$$

or in the form of operator equation

$$
\begin{aligned}
B_{0} M_{1}= & -\mathcal{P}_{Y} l \int_{0}^{\cdot} e^{(\cdot-\tau) A} D(\tau) \overline{G\left[D(\cdot) \bar{X}_{0}\left(\cdot, \bar{M}_{0}\right)\right]}(\tau) \times \\
& \times e^{(\tau-\cdot) A} d \tau-\mathcal{P}_{Y} l \int_{0}^{\cdot} e^{(\cdot-\tau) A} D(\tau) \times \\
& \times \overline{G\left[D(\cdot) \bar{Y}_{0}(\cdot)\left[P_{N\left(B_{0}\right)} M_{\rho}\right]\right]}(\tau) e^{(\tau-\cdot) A} d \tau .
\end{aligned}
$$

By virtue of solvability, we find the operator

$$
M_{1}=\bar{M}_{1}+\mathcal{F}_{1}\left[P_{N\left(B_{0}\right)} M_{\rho}\right]
$$

where

$$
\begin{gathered}
\bar{M}_{1}=-B_{0}^{-} \mathcal{P}_{Y} l \int_{0}^{\cdot} e^{(\cdot-\tau) A} D(\tau) \overline{G\left[D(\cdot) \bar{X}_{0}\left(\cdot, \bar{M}_{0}\right)\right]}(\tau) \times \\
\times e^{(\tau-\cdot) A} d \tau, \\
\mathcal{F}_{1}\left[P_{N\left(B_{0}\right)} M_{\rho}\right]=P_{N\left(B_{0}\right)} M_{\rho}- \\
-B_{0}^{-} \mathcal{P}_{Y} l \int_{0}^{\cdot} e^{(\cdot-\tau) A} D(\tau) \overline{G\left[D(\cdot) \bar{Y}_{0}(\cdot)\left[P_{N\left(B_{0}\right)} M_{\rho}\right]\right]}(\tau) \times \\
\times e^{(\tau-\cdot) A} d \tau .
\end{gathered}
$$

Then the set of solutions of boundary value problem (21), (22) has the form

$X_{1}\left(t, M_{\rho}\right)=\bar{X}_{1}\left(t, \bar{M}_{1}\right)+\bar{Y}_{1}(t)\left[P_{N\left(B_{0}\right)} M_{\rho}\right], \quad M_{\rho} \in \mathcal{L}(H)$

where

$$
\begin{aligned}
& \bar{X}_{1}\left(t, \bar{M}_{1}\right)=e^{t A} P_{N(Q)} \bar{M}_{1} e^{-t A}+\overline{\left(G\left[D(\cdot) \bar{X}_{0}\left(\cdot, \bar{M}_{0}\right)\right]\right)}(t), \\
& \bar{Y}_{1}(t)[R]:=\overline{\left(G\left[D(\cdot) \bar{Y}_{0}(\cdot)[R]\right]\right)}(t)+e^{t A} P_{N(Q)} \mathcal{F}_{1}[R] e^{-t A} .
\end{aligned}
$$

Hence the solution set of the boundary value problem (26), (27) has the form

$$
X_{2}\left(t, M_{2}\right)=e^{t A} P_{N(Q)} M_{2} e^{-t A}+\overline{\left(G\left[D(\cdot) X_{1}\left(\cdot, M_{\rho}\right)\right]\right)}(t),
$$

or in the from

$$
\begin{aligned}
X_{2}\left(t, M_{2}\right)= & e^{t A} P_{N(Q)} M_{2} e^{-t A}+\overline{\left(G\left[D(\cdot) \bar{X}_{1}\left(\cdot, \bar{M}_{1}\right)\right]\right)}(t)+ \\
& +\overline{\left(G\left[D(\cdot) \bar{Y}_{1}(\cdot)\left[P_{N\left(B_{0}\right)} M_{\rho}\right]\right]\right)}(t) .
\end{aligned}
$$

Arguing further by induction, one can readily show that if the condition on the product of projections is satisfied, then the problem of determining the coefficient $X_{i}(t)$ of $\varepsilon^{i}$ in the series (20) is reduced to the solvability of the operator boundary value problem

$$
\begin{gathered}
\frac{d X_{i}(t)}{d t}=\left[A, X_{i}(t)\right]+D(t) X_{i-1}\left(t, M_{i-1}\right), \\
l X_{i}(\cdot)=0 .
\end{gathered}
$$

The operator $M_{i}$ is determined as follows:

$$
M_{i}=\bar{M}_{i}+\mathcal{F}_{i}\left[P_{N\left(B_{0}\right)} M_{\rho}\right],
$$


where

$$
\begin{gathered}
\bar{M}_{i}=-B_{0}^{-} \mathcal{P}_{Y} l \int_{0}^{\cdot} e^{(\cdot-\tau) A} D(\tau) \times \\
\times \overline{G\left[D(\cdot) \bar{X}_{i-1}\left(\cdot, \bar{M}_{i-1}\right)\right]}(\tau) e^{(\tau-\cdot) A} d \tau, \\
\mathcal{F}_{i}\left[P_{N\left(B_{0}\right)} M_{\rho}\right]=P_{N\left(B_{0}\right)} M_{\rho}- \\
-B_{0}^{-} \mathcal{P}_{Y} l \int_{0}^{\cdot} e^{(\cdot-\tau) A} D(\tau) \times
\end{gathered}
$$$$
\times \overline{G\left[D(\cdot) \bar{Y}_{i-1}(\cdot)\left[P_{N\left(B_{0}\right)} M_{\rho}\right]\right]}(\tau) e^{(\tau-\cdot) A} d \tau,
$$

and then the set of solutions of the boundary value problem (30), (31) can be represented in the form

$$
X_{i}\left(t, M_{\rho}\right)=\bar{X}_{i}\left(t, \bar{M}_{i}\right)+\bar{Y}_{i}(t)\left[P_{N\left(B_{0}\right)} M_{\rho}\right], \quad M_{\rho} \in \mathcal{L}(H)
$$

where

$$
\begin{aligned}
& \left.\bar{X}_{i}\left(t, \bar{M}_{i}\right)=e^{t A} P_{N(Q)} \bar{M}_{i} e^{-t A}+\overline{\left(G\left[D(\cdot) \bar{X}_{i-1}\left(\cdot, \bar{M}_{i-1}\right)\right]\right.}\right)(t) \\
& \bar{Y}_{i}(t)[R]:=\overline{\left(G\left[D(\cdot) \bar{Y}_{i-1}(\cdot)[R]\right]\right)}(t)+e^{t A} P_{N(Q)} \mathcal{F}_{i}[R] e^{-t A} .
\end{aligned}
$$

The convergence of the series (20) for given $\varepsilon$ can be proved by the standard majorant method as in [22]. Thus, the following theorem holds.

Theorem 2. If the unperturbed operator boundary value problem (3), (4) has classical solutions, then, under the condition

$$
\mathcal{P}_{N\left(B_{0}^{*}\right)} \mathcal{P}_{Y}=0
$$

the operator boundary value problem (1), (2) has a $\rho$ parameter family of classical solutions in the form of the series

$$
X_{i}\left(t, \varepsilon, M_{\rho}\right)=\sum_{i=0}^{+\infty} \varepsilon^{i}\left[\bar{X}_{i}\left(t, \bar{M}_{i}\right)+\bar{Y}_{i}(t)\left[P_{N\left(B_{0}\right)} M_{\rho}\right]\right]
$$

for any $M_{\rho} \in \mathcal{L}(H)$, which is absolutely convergent for sufficiently small given $\varepsilon \in\left(0, \varepsilon_{*}\right]$; here

$$
\begin{gathered}
X_{0}\left(t, M_{\rho}\right)=\bar{X}_{0}\left(t, \bar{M}_{0}\right)+\bar{Y}_{0}(t)\left[P_{N\left(B_{0}\right)} M_{\rho}\right], M_{\rho} \in \mathcal{L}(H) \\
\bar{X}_{0}\left(t, \bar{M}_{0}\right)=e^{t A} P_{N(Q)} \bar{M}_{0} e^{-t A}+e^{t A} \bar{Q}-\alpha e^{-t A}+\overline{G[\Phi]}(t) \\
\bar{Y}_{0}(t)\left[P_{N\left(B_{0}\right)} M_{\rho}\right]:=e^{t A} P_{N(Q)} P_{N\left(B_{0}\right)} M_{\rho} e^{-t A} \\
\bar{X}_{i}\left(t, \bar{M}_{i}\right)=e^{t A} P_{N(Q)} \bar{M}_{i} e^{-t A}+\overline{\left(G\left[D(\cdot) \bar{X}_{i-1}\left(\cdot, \bar{M}_{i-1}\right)\right]\right.}(t) \\
\bar{Y}_{i}(t)\left[P_{N\left(B_{0}\right)} M_{\rho}\right]:=\overline{\left(G\left[D(\cdot) \bar{Y}_{i-1}(\cdot)\left[P_{N\left(B_{0}\right)} M_{\rho}\right]\right]\right)}(t)+ \\
+e^{t A} P_{N(Q)} \mathcal{F}_{i}\left[P_{N\left(B_{0}\right)} M_{\rho}\right] e^{-t A}, i=1,2, \ldots . \\
\mathcal{F}_{i}\left[P_{N\left(B_{0}\right)} M_{\rho}\right]=P_{N\left(B_{0}\right)} M_{\rho}- \\
-B_{0}^{-} \mathcal{P}_{Y} l \int_{0} e^{(\cdot-\tau) A} D(\tau) \times
\end{gathered}
$$$$
\times \overline{G\left[D(\cdot) \bar{Y}_{i-1}(\cdot)\left[P_{N\left(B_{0}\right)} M_{\rho}\right]\right]}(\tau) e^{(\tau-\cdot) A} d \tau .
$$

\section{Conclusions}

1. Proposed in the article method works in the following case: assume that unperturbed boundary value problem (5), (6) does not have strong generalized (or classical solutions), i.e. condition (10) is satisfied. In this case we seek solutions of the boundary value problem (1), (2) in the form of part of the series

$$
X(t, \varepsilon)=\sum_{i=-k}^{+\infty} \varepsilon^{i} X_{i}(t)
$$

2. Number of solutions of boundary value problem (1), (2) depends from the dimension of the subspace $P_{N\left(B_{0}\right)} \mathcal{L}(H)$.

3 . We can use the modification of theorem 1 and 2 for investigating the following boundary value problem

$$
\frac{d X(t, \varepsilon)}{d t}=[A(t), X(t, \varepsilon)]+\Phi(t)+\varepsilon D(t) X(t, \varepsilon), t \in J
$$

$$
l X(\cdot, \varepsilon)=\alpha,
$$

with nonstationary operator-valued function $A(t)$ which can be unbounded in general case [23].

4. Proposed in the article method can be used for finding of bounded on the whole axis solutions. Namely, consider operator differential equation

$$
\frac{d X(t)}{d t}=[A(t), X(t)]+\Phi(t)
$$

where $t \in R, \Phi(t) \in B C(R, \mathcal{L}(H)), B C(R, \mathcal{L}(H))$ is the Banach space of continuous and bounded on $R$ operator-valued functions:

$B C(R, H):=\left\{\Phi: R \rightarrow H,\|\Phi\|_{\mathcal{L}(H)}=\sup _{t \in R}\|\Phi(t)\|<\infty\right\} ;$

$A(t) \in \mathcal{L}(H), t \in R:$

$$
\|A\|\left\|=\sup _{t \in R}\right\| A(t) \|<\infty
$$

and homogeneous equation

$$
\frac{d X(t)}{d t}=[A(t), X(t)]
$$

We say that evolution operator $U(t)$ is defined on (35) if the following equality is hold:

$$
\frac{d U(t)}{d t}=[A(t), U(t)], U(0)=I
$$

Definition. [15], [24]. Equation (35) admits an exponential dichotomy on the interval $J$ if there exist a projector $P\left(P^{2}=\right.$ $P$ ) and constants $K \geq 1$ and $\alpha>0$ such that, for any $t, s \in J$, the following estimates are true:

$$
\begin{gathered}
\left\|U(t) P U^{-1}(s)\right\| \leq K e^{-\alpha(t-s)}, t \geq s, \\
\left\|U(t)(E-P) U^{-1}(s)\right\| \leq K e^{\alpha(t-s)}, s \geq t,
\end{gathered}
$$

where $U(t)=U(t, 0)$ is the evolution operator of equation (35). As in [23] we can prove the following theorem. 
Exponential Dichotomy and Bifurcation Conditions of Solutions of the Hamiltonian Operators Boundary Value Problems in the Hilbert Space

Theorem 3. Suppose that the homogeneous equation (35) admits an exponential dichotomy on the semiaxes $R_{+}$and $R_{-}$ with projectors $P$ and $Q$ and constants $K_{1}, \alpha_{1}$ and $K_{2}, \alpha_{2}$, respectively. If the operator

$$
D=P-(E-Q): \mathcal{L}(H) \rightarrow \mathcal{L}(H)
$$

which acts from the Banach space $\mathcal{L}(H)$ into itself, is generalized invertible, then the following assertions are true:

(i) in order that solutions of equation (34) bounded on the entire real axis exist, it is necessary and sufficient that the operator-valued function $\Phi(t) \in B C(R, \mathcal{L}(H))$ satisfies the condition

$$
\int_{-\infty}^{+\infty} H(t) \Phi(t) d t=0 ;
$$

(ii) under condition (37), solutions of the equation (34) bounded on the entire axis have the form

$$
X(t, M)=U(t) P P_{N(D)} M+(G[\Phi])(t), M \in \mathcal{L}(H),
$$

where

$$
(G[f])(t)=U(t)\left\{\begin{array}{c}
\int_{0}^{t} P U^{-1}(s) \Phi(s) d s- \\
-\int_{t}^{+\infty}(E-P) U^{-1}(s) \Phi(s) d s+ \\
+P D^{-}\left(\int_{0}^{+\infty}(E-P) U^{-1}(s) \Phi(s) d s\right. \\
\left.+\int_{-\infty}^{0} Q U-1(s) \Phi(s) d s\right), t \geq 0, \\
\int_{-\infty}^{t} Q U^{-1}(s) \Phi(s) d s- \\
-\int_{t}^{0}(E-Q) U^{-1}(s) \Phi(s) d s+ \\
(E-Q) D^{-}\left(\int_{0}^{+\infty}(E-P) U^{-1}(s) \Phi(s) d s\right. \\
+\int_{-\infty}^{0} Q U^{-1}(s) \Phi(s) d s, t \leq 0
\end{array}\right.
$$

is the generalized Green operator of the problem of solutions bounded on the entire axis $R$ and possessing the following properties:

$$
\begin{gathered}
(G[\Phi])(0+0)-(G[\Phi])(0-0)=-\int_{-\infty}^{+\infty} H(t) \Phi(t) d t=0, \\
(L G[\Phi])(t)=\Phi(t), t \in R, \\
L X(t):=\frac{d X(t)}{d t}-[A(t), X(t)],
\end{gathered}
$$

where $H(t)=P_{N\left(D^{*}\right)} Q U^{-1}(t)=P_{N\left(D^{*}\right)}(I-P) U^{-1}(t)$, $D^{-}$- is the generalized inverse of the operator $D, P_{N(D)}$ and $P_{N\left(D^{*}\right)}$ are the projectors that project $\mathcal{L}(H)$ onto the kernel $N(D)$ and the cokernel $N\left(D^{*}\right)$ of the operator $D$, respectively.

Remark. Now we show convergence one of the infinite integrals:

$$
\begin{gathered}
\\
\left\|U(t) \int_{t}^{+\infty}(E-P) U^{-1}(s) \Phi(s) d s\right\| \leq \\
\leq \int_{t}^{+\infty}\left\|U(t)(E-P) U^{-1}(s) \Phi(s)\right\| d s \leq \\
\leq\|\Phi\|_{\mathcal{L}(H)} \int_{t}^{+\infty}\left\|U(t)(E-P) U^{-1}(s)\right\| d s
\end{gathered}
$$

$$
\begin{gathered}
\leq\|\Phi\|_{\mathcal{L}(H)} \int_{t}^{+\infty} K_{1} e^{\alpha_{1}(t-s)} d s= \\
=\left.\|\Phi\|_{\mathcal{L}(H)} K_{1} e^{\alpha_{1} t} \frac{e^{-\alpha_{1} s}}{-\alpha_{1}}\right|_{t} ^{+\infty}= \\
=\|\Phi\|_{\mathcal{L}(H)} \frac{K_{1}}{\alpha_{1}} .
\end{gathered}
$$

\section{REFERENCES}

[1] Bogolyubov N.N., and MitropolŁkii Yu.A. Asimptoticheskie metody $\mathrm{v}$ teorii nelineinykh kolebanii (Asymptotic Methods in the Theory of Nonlinear Oscillations), Gostekhteorizdat, Moscow, 1955.

[2] Maslov V.P. Asimptoticheskie metody i teoriya vozmushchenii (Asymptotic Methods and Perturbation Theory), Nauka, Moscow, 1988.

[3] Arnold V.I., Afraimovich V.S., IlŁashenko Yu.S., and ShilŁikov L.P. Teoriya bifurkatsii (Bifurcation Theory), VINITI, Moscow, 1980 .

[4] De la Llave R. A tutorial on KAM theory, Proceedings of Symposia in Pure Mathematics, Providence: Amer. Math. Soc., vol. 69, 175-?96, 2001.

[5] Maslov V.P. Operatornye metody (Operator Methods), Nauka, Moscow, 1973.

[6] Nayfeh A.H. Perturbation Methods, Wiley, New York, 1973.

[7] Grebennikov E.A. and Ryabov Yu.A. Konstruktivnye metody analiza nelineinykh sistem (Constructive Methods for the Analysis of Nonlinear Systems), Nauka, Moscow, 1979.

[8] Treschev D., Zubelevich O. Introduction to the perturbation theory of Hamiltonian systems, Springer-Verlag, Berlin, Heidelberg, 2010.

[9] Moore E. H. On the Reciprocal of the General Algebraic Matrix (Abstract), Bull. Amer. Math. Soc., v.26, 394-395, 1920.

[10] Penrose R. A. Generalized Inverse for Matrices, Proc. Cambridge Philos. Soc., vol. 51, 406 - 413, 1955.

[11] Boichuk A.A., and Samoilenko A.M. Generalized Inverse Operators and Fredholm Boundary Value Problems, VSP, Utrecht, 2004.

[12] Korolyuk V.S. and Turbin A.F. Matematicheskie osnovy fazovogo ukrupneniya slozhnykh sistem (Mathematical Foundations of the Phase Lumping of Complex Systems), Naukova Dumka, Kiev, 1978.

[13] Pokutnyi O.O. Generalized-invertible operator in the Banach Hilbert and Frechet spaces, Visnik of the Kiev National Taras Shevchenko university, Series of physical and mathematical sciences, No. 4, 167-171, 2013.

[14] Boichuk O. A., Krivosheya S. A. Criterion for the solvability of matrix equations of the Lyapunov type, Ukr. Mat. Zh., 50, No. 8, 1162-?169, 1998. 
[15] Krein M.G., Daletskiy Yu. L. Ustoichivost' resheniy differencial'nih uravneniy $\mathrm{v}$ banahovom prostranstve (Stability of solutions of differential equations in the Banach space), Nauka, Moscow, 1979.

[16] Tikhonov A.N. and Arsenin V.Ya. Metody resheniya nekorrektnykh zadach (Methods for Solving Ill Posed Problems), Nauka, Moscow, 1986.

[17] Vishik M.I., Lyusternik L.A. The solutions of some perturbation problems for matrices and selfadjoint or non-selfadjoint differential equations I, Russian Mathematical Surveys, vol. 15, No. 3, 3-80, 1960.

[18] Panasenko E. V., Pokutnyi O. O. Boundary-Value Problems for the Lyapunov Equation in Banach Spaces, Journal of mathematical sciences, v. 223, 1-7, 2017.

[19] Pokutnyi O.O. Boundary value problem for an operatordifferential Riccati Equation in the Hilbert space on the interval, Advances in Pure Mathematics, 5, 865-873, 2015.
[20] Boichuk A.A., Pokutnyi A.A. Perturbation theory of operator equations in th Frechet and Hilbert spaces, Ukrainian mathematical journal, vol. 67, No. 9, 1327-1335, 2016.

[21] Lyashko S.I., Nomirovskii D.A., Petunin Yu.I., and Semenov V.V., Dvadtsataya problema Gil Łerta. Obobshchennye resheniya operatornykh uravnenii (HilbertŁ Twentieth Problem. Generalized Solutions of Operator Equations), VilŁams, Moscow, 2009.

[22] Boichuk A.A. and Pokutnij A.A. Bounded solutions of linear perturbed differential equations in a Banach space, Tatra Mountains Math. Publ., vol. 38, 29-?1, 2007.

[23] Pokutnyi O.A. Bounded solutions of linear and weakly nonlinear differential equations in Banach space with unbounded linear part, Differential equations, v. 48, No. 6, 803-?13, 2012.

[24] Palmer K. J. Exponential dichotomies and transversal homoclinic points, J.Diff. Eq., vol. 55, 225-256, 1984. 